\title{
THE LOCAL FORM OF DOUBLY STOCHASTIC MAPS AND JOINT MAJORIZATION IN II 1 FACTORS
}

\author{
MARTÍN ARGERAMI AND PEDRO MASSEY
}

\begin{abstract}
We find a description of the restriction of doubly stochastic maps to separable abelian $C^{*}$-subalgebras of a $\mathrm{II}_{1}$ factor $\mathcal{M}$. We use this local form of doubly stochastic maps to develop a notion of joint majorization between $n$ tuples of mutually commuting self-adjoint operators that extends those of Kamei (for single self-adjoint operators) and Hiai (for single normal operators) in the $\mathrm{II}_{1}$ factor case. Several characterizations of this joint majorization are obtained. As a byproduct we prove that any separable abelian $C^{*}$-subalgebra of $\mathcal{M}$ can be embedded into a separable abelian $C^{*}$-subalgebra of $\mathcal{M}$ with diffuse spectral measure.
\end{abstract}

Keywords: Joint majorization, doubly stochastic map, convex hull, unitary orbit

\section{INTRODUCTION}

Majorization between self-adjoint operators in finite factors was introduced by Kamei [21] as an extension of Ando's definition of majorization between self-adjoint matrices [4]. Later on, Hiai considered majorization in semifinite factors between self-adjoint and normal operators $[15,16]$. The reason why majorization has attracted the attention of many researchers (see the discussion in [16] and the references therein) is that it provides a rather subtle way to compare operators that occurs naturally in many contexts (for example $[5,13,14]$ ). Recently, majorization has regained interest because of its relation with norm-closed unitary orbits of selfadjoint operators and conditional expectations onto abelian subalgebras $[5,6,10,14$, $18,19,24,26]$. One of the goals of this paper (section 4) is to obtain an extension of the notion of majorization between normal operators to that of joint majorization between $n$-tuples of commuting self-adjoint operators in a $\mathrm{II}_{1}$ factor (such extension is achieved in [22] for finite dimensional factors). In order to obtain characterizations of this extended notion we describe the local form of a doubly stochastic (DS) map: we get a family of particularly well behaved DS maps that approximate the restriction of any DS map to separable abelian $C^{*}$-subalgebras of the $\mathrm{II}_{1}$ factor (section 3). As a byproduct, we construct separable abelian diffuse refinements of separable abelian $C^{*}$-subalgebras of a $\mathrm{II}_{1}$ factor $\mathcal{M}$. This construction could have interest on its own; it has been developed in [23], and similar ideas have been used in [7,8]. Some of the techniques we use appear to be new, even in the single element case.

The paper is organized as follows. In section 2 we recall some facts about abelian $C^{*}$-subalgebras of a $\mathrm{II}_{1}$ factor. In section 3 , after describing some technical results, we obtain a description of the local structure of doubly stochastic maps. In section 4 we introduce and develop the notion of joint majorization between finite abelian

Supported in part by the Natural Sciences and Engineering Research Council of Canada. 2000 Mathematics Subject Classification: Primary 46L51; Secondary 46L10 . 
families of self-adjoint operators in a $\mathrm{II}_{1}$ factor and we obtain several characterizations of this relation. Section 5 deals with joint unitary orbits of abelian families. Finally, in section 6 we prove the results described in section 3 .

\section{Preliminaries}

Throughout the paper $\mathcal{M}$ will be a $\mathrm{II}_{1}$ factor with normalized faithful normal trace $\tau$. The $C^{*}$-subalgebras of $\mathcal{M}$ are always assumed unital. The subspace of self-adjoint elements of $\mathcal{M}$ will be denoted by $\mathcal{M}_{s a}$. An abelian family $\left(a_{i}\right)_{i=1}^{n}$ in $\mathcal{M}_{s a}$, is a finite family of mutually commuting self-adjoint operators in $\mathcal{M}$. If $\left(a_{i}\right)_{i=1}^{n} \subseteq \mathcal{M}_{s a}$ is an abelian family then $C^{*}\left(a_{1}, \ldots, a_{n}\right)$ denotes the (unital) separable abelian $C^{*}$-subalgebra of $\mathcal{M}$ generated by $a_{1}, \ldots, a_{n}$. If $\mathcal{A}$ is an arbitrary abelian $C^{*}$-subalgebra of $\mathcal{M}$ then $\Gamma(\mathcal{A})$ denotes its space of characters, i.e. the set of nonzero *-homomorphisms $\gamma: \mathcal{A} \rightarrow \mathbb{C}$ endowed with the weak*-topology. It is well-known that the set $\Gamma(\mathcal{A})$ is a compact space and that $\mathcal{A} \simeq C(\Gamma(\mathcal{A}))$, where $C(\Gamma(\mathcal{A}))$ denotes the $C^{*}$-algebra of continuous functions on $\Gamma(\mathcal{A})$. We will use 1 to denote the constant function and $\pi_{i}: \mathbb{R}^{n} \rightarrow \mathbb{R}$ to denote the projection onto the $i^{t h}$ coordinate.

2.1. Joint spectral measures and joint spectral distributions. As we will consider a several-variable extension of continuous functional calculus, we state a few facts about it (a different description can be found in [27]). Let $\bar{a}=\left(a_{i}\right)_{i=1}^{n}$ be an abelian family in $\mathcal{M}_{s a}$. If $\mathcal{A}=C^{*}\left(a_{1}, \ldots, a_{n}\right)$, then $\Gamma(\mathcal{A})$ can be embedded in $\prod_{i=1}^{n} \sigma\left(a_{i}\right) \subseteq \mathbb{R}^{n}$. Indeed, the map $\Phi: \Gamma(\mathcal{A}) \rightarrow \prod_{i=1}^{n} \sigma\left(a_{i}\right) \subseteq \mathbb{R}^{n}$ given by $\Phi(\gamma)=$ $\left(\gamma\left(a_{1}\right), \ldots, \gamma\left(a_{n}\right)\right)$ is a continuous injection and therefore $\Gamma(\mathcal{A})$ is homeomorphic to its image under this map; this image is called the joint spectrum of the family and we denote it by $\sigma(\bar{a})$. Note that $\mathcal{A} \simeq C(\sigma(\bar{a}))$ as $C^{*}$-algebras, and so for each $f \in C(\sigma(\bar{a}))$ there exists a normal operator, denoted $f\left(a_{1}, \ldots, a_{n}\right)$, that corresponds to $f$ under the isomorphism.

If $\mathcal{A} \subseteq \mathcal{M}$ is a separable $C^{*}$-subalgebra then $\Gamma(\mathcal{A})$ is metrizable and the representation $C(\Gamma(\mathcal{A})) \simeq \mathcal{A} \subseteq \mathcal{M}$ induces a spectral measure $E_{\mathcal{A}}$ [12, IX.1.14] that takes values on the lattice $\mathcal{P}(\mathcal{M})$ of projections of $\mathcal{M}$. Let $\mu_{\mathcal{A}}$ be the (scalar) regular Borel measure on $\Gamma(\mathcal{A})$ defined by

$$
\mu_{\mathcal{A}}(\Delta)=\tau\left(E_{\mathcal{A}}(\Delta)\right)
$$

The regularity of $\mu_{\mathcal{A}}$ follows from the fact that every open set is $\sigma$-compact $[25$, 2.18]. The map $\Lambda: L^{\infty}\left(\Gamma(\mathcal{A}), \mu_{\mathcal{A}}\right) \rightarrow \mathcal{M}$ given by $\Lambda(h)=\int_{\Gamma(\mathcal{A})} h d E_{\mathcal{A}}$ is a normal *-monomorphism (note that in this case the weak* topology of $L^{\infty}\left(\Gamma(\mathcal{A}), \mu_{\mathcal{A}}\right)$, restricted to the unit ball, is metrizable) and we have

$$
\tau(\Lambda(h))=\int_{\Gamma(\mathcal{A})} h d \mu_{\mathcal{A}}, \quad \forall h \in L^{\infty}\left(\Gamma(\mathcal{A}), \mu_{\mathcal{A}}\right) .
$$

We will consider the von Neumann algebra $L^{\infty}(\mathcal{A}):=\Lambda\left(L^{\infty}\left(\Gamma(\mathcal{A}), \mu_{\mathcal{A}}\right)\right) \subseteq \mathcal{M}$.

When $\bar{a}=\left(a_{i}\right)_{i=1}^{n}$ and $\mathcal{A}=C^{*}\left(a_{1}, \ldots, a_{n}\right)$, we call $E_{\bar{a}}:=E_{\mathcal{A}}$ and $\mu_{\bar{a}}:=\mu_{\mathcal{A}}$ are the joint spectral measure and joint spectral distribution of the abelian family $\bar{a}$ and we denote by $\Lambda_{\bar{a}}: L^{\infty}\left(\Gamma(\bar{a}), \mu_{\bar{a}}\right) \rightarrow L^{\infty}(\mathcal{A})$ the normal isomorphism defined above. It is straightforward to verify that $\Lambda_{\bar{a}}\left(\pi_{i}\right)=a_{i}, 1 \leq i \leq n$. Recall that for each $h \in L^{\infty}\left(\Gamma(\bar{a}), \mu_{\bar{a}}\right)$ we write $h\left(a_{1}, \ldots, a_{n}\right)$ for the operator $\Lambda_{\bar{a}}(h) \in \mathcal{A}$. In the 
case of a single self-adjoint operator $a \in \mathcal{M}_{s a}$ the measure $\mu_{a}$ is the usual spectral distribution of $a[10]$.

In the particular case when $x \in \mathcal{M}$ is a normal operator, the real and imaginary parts of $x$ are mutually commuting self-adjoint elements of $\mathcal{M}$. Identifying the complex plane with $\mathbb{R}^{2}$ in the usual way, the spectrum of $x$ coincides with the joint spectrum of the abelian pair $(\operatorname{Re}(x), \operatorname{Im}(x))$, and that the spectral measure of $x$ coincides with the joint spectral measure of $(\operatorname{Re}(x), \operatorname{Im}(x))$.

2.2. Comparison of measures and diffuse measures. We denote by $M_{+}^{\sim}\left(\mathbb{R}^{n}\right)$ the set of all regular finite positive Borel measures $\nu$ on $\mathbb{R}^{n}$ with $\int\|\zeta\| d \nu(\zeta)<\infty$. We write $\nu(f)=\int_{\mathbb{R}^{n}} f d \nu$, for every $\nu \in M_{+}^{\sim}\left(\mathbb{R}^{n}\right)$ and every $\nu$-integrable function $f$.

Definition 2.1. Let $\mu, \nu \in M_{+}^{\sim}\left(\mathbb{R}^{n}\right)$.

(1) We write $\nu \sim \mu$ whenever $\nu(1)=\mu(1)$ and $\nu\left(\pi_{j}\right)=\mu\left(\pi_{j}\right)$ for every $1 \leq j \leq$ $n$;

(2) we say that $\mu$ is majorized by $\nu$, and we write $\mu \prec \nu$, if for every $\mu_{1}, \ldots, \mu_{m} \in$ $M_{+}^{\sim}\left(\mathbb{R}^{n}\right)$ with $\sum_{i=1}^{m} \mu_{i}=\mu$ there exist $\nu_{1}, \ldots, \nu_{m} \in M_{+}^{\sim}\left(\mathbb{R}^{n}\right)$ such that $\sum_{i=1}^{m} \nu_{i}=\nu$, and $\nu_{i} \sim \mu_{i}, 1 \leq j \leq n$.

The relation $\prec$ in Definition 2.1 does not seem to be called "majorization" in the literature, but it will be a suitable name for us in the light of Theorem 4.5.

Theorem 2.2. [3, I.3.2] Let $\mu, \nu \in M_{+}^{\sim}\left(\mathbb{R}^{n}\right)$. Then $\mu \prec \nu$ if and only if $\mu(f) \leq \nu(f)$ for every continuous convex function $f: \mathbb{R}^{n} \rightarrow \mathbb{R}$. (1).

The next corollary is an immediate consequence of Theorem 2.2 and the identity

Corollary 2.3. Let $\bar{a}=\left(a_{i}\right)_{i=1}^{n}, \bar{b}=\left(b_{i}\right)_{i=1}^{n} \subset \mathcal{M}_{s a}$ be two abelian families. Then $\mu_{\bar{a}} \prec \mu_{\bar{b}}$ if and only if $\tau\left(f\left(a_{1}, \ldots, a_{n}\right)\right) \leq \tau\left(f\left(b_{1}, \ldots, b_{n}\right)\right)$ for every continuous convex function $f: \mathbb{R}^{n} \rightarrow \mathbb{R}$.

We end this section with the following elementary fact about diffuse (scalar) measures, i.e. measures without atoms (recall that $x$ is an atom of a measure $\mu$ if $\mu(\{x\})>0)$.

Lemma 2.4. Let $K \subset \mathbb{R}^{n}$ be compact and let $\mu$ be a regular diffuse Borel probability measure on $K$. Then for every $\alpha \in(0,1)$ there exists a measurable set $S \subset K$ such that $\mu(S)=\alpha$.

\section{THE LOCAL FORM OF DOUBLY STOCHASTIC MAPS}

This section deals with doubly stochastic maps (see Definition 3.1 below). These maps play an important role in the theory of majorization between self-adjoint operators (see for instance $[1,2,15,16]$ ); they will also play a central role in majorization between abelian families (section 4).

For the sake of clarity, several proofs of technical results from this section will be delayed until section 6 .

Let $\mathcal{A} \subseteq \mathcal{M}$ be an abelian $C^{*}$-subalgebra, and let $E_{\mathcal{A}}$ and $\mu_{\mathcal{A}}$ denote the spectral measure and the spectral distribution of $\mathcal{A}$ as defined in section 2.1.

\section{Definition 3.1.}


(1) A linear map $\Phi: \mathcal{M} \rightarrow \mathcal{M}$ is said to be doubly stochastic [15] if it is unital, positive, and trace preserving. The set of all doubly stochastic maps on $\mathcal{M}$ is denoted by by $D S(\mathcal{M})$;

(2) If $x \in \Gamma(\mathcal{A})$ is such that $E_{\mathcal{A}}(\{x\}) \neq 0$, we say that $x$ is an atom for $E_{\mathcal{A}}$;

(3) the set of atoms of $E_{\mathcal{A}}$ is denoted $\operatorname{At}\left(E_{\mathcal{A}}\right)$;

(4) we say that $\mathcal{A}$ is diffuse if $A t\left(E_{\mathcal{A}}\right)=\emptyset$;

(5) the set $\mathcal{D}(\mathcal{M})$ is the convex semigroup $\mathcal{D}(\mathcal{M})=\operatorname{conv}\{A d u: u \in \mathcal{U}(\mathcal{M})\}$.

Since $\mu_{\mathcal{A}}=\tau \circ E_{\mathcal{A}}$, the faithfulness of the trace implies that $\operatorname{At}\left(\mu_{\mathcal{A}}\right)=\operatorname{At}\left(E_{\mathcal{A}}\right)$. The following theorem states that spectral measures of a separable $\mathcal{A}$ can be refined in a coherent way.

Theorem 3.2. Let $\mathcal{A} \subseteq \mathcal{M}$ be a separable abelian $C^{*}$-subalgebra. Then there exists $a \in \mathcal{M}_{\text {sa }}$ such that $C^{*}(\mathcal{A}, a)$ is abelian and diffuse.

Proof. (see section 6).

Since the atoms of $E_{\mathcal{A}}$ are in correspondence with the set of minimal projections of $L^{\infty}(\mathcal{A})$, Theorem 3.2 provides a way to embed $\mathcal{A}$ into a separable $C^{*}$-subalgebra $\tilde{\mathcal{A}}=C^{*}(\mathcal{A}, a)$ such that $L^{\infty}(\tilde{\mathcal{A}})$ has no minimal projections (see Remark 6.3 for further discussion).

Any operator in a von Neumann algebra can be approximated in norm by linear combinations of projections. In the case of a $\mathrm{II}_{1}$ factor, an added requirement could be for the projections to have equal trace; with such requirement, the norm approximation is usually lost, and only norm- 1 and norm- 2 approximation can be achieved. What Proposition 3.3 shows is that norm approximation can still be obtained by taking linear combinations of partitions of the unity (that is, projections with equal trace).

Proposition 3.3. Let $\mathcal{B} \subset \mathcal{M}$ be a separable, diffuse, abelian $C^{*}$-subalgebra. Then there is an unbounded set $\mathbb{M} \subseteq \mathbb{N}$ such that for every $m \in \mathbb{M}$ there exist $k=k(m)$ partitions of the unity $\left\{q_{i}^{t, m}\right\}_{i=1}^{m} \subseteq \mathcal{B}^{\prime} \cap \mathcal{M}, 1 \leq t \leq k$, with $\tau\left(q_{i}^{t, m}\right)=1 / m(1 \leq i \leq$ $m, 1 \leq t \leq k)$, and such that for each $b \in \mathcal{B}$,

$$
\lim _{m \rightarrow \infty}\left\|b-\frac{1}{k} \sum_{t=1}^{k}\left(\sum_{i=1}^{m} \beta_{i}^{t, m} q_{i}^{t, m}\right)\right\|=0,
$$

where $\beta_{i}^{t, m}=m \tau\left(b q_{i}^{t, m}\right)$.

Proof. (see section 6).

Remark 3.4. For fixed $m$ and partitions of the unity $\left\{q_{i}^{t}\right\}_{i=1}^{m}, 1 \leq t \leq k$, the linear map

$$
b \mapsto \frac{1}{k} \sum_{t=1}^{k}\left(\sum_{i=1}^{m} m \tau\left(b q_{i}^{t}\right) q_{i}^{t}\right)
$$

is a contraction with respect to the operator norm. 
Lemma 3.5. Let $\left\{p_{i}\right\}_{i=1}^{m},\left\{q_{i}\right\}_{i=1}^{m} \subseteq \mathcal{M}$ be partitions of the unity such that $\tau\left(p_{i}\right)=$ $\tau\left(q_{i}\right)=\frac{1}{m}$, and let $T \in D S(\mathcal{M})$. Let $\beta_{1}, \ldots, \beta_{m} \in \mathbb{R}$ and $\alpha_{i}=m \sum_{j=1}^{m} \beta_{j} \tau\left(T\left(q_{j}\right) p_{i}\right)$, $1 \leq i \leq m$. Then there exists $\rho \in \mathcal{D}(\mathcal{M})$ such that

$$
\sum_{i=1}^{m} \alpha_{i} p_{i}=\rho\left(\sum_{i=1}^{m} \beta_{i} q_{i}\right)
$$

Proof. Let $\gamma_{i, j}=m \tau\left(T\left(q_{j}\right) p_{i}\right) \geq 0$; it is then straightforward to verify that $\gamma=$ $\left(\gamma_{i, j}\right) \in \mathbb{R}^{m \times m}$ is a doubly stochastic matrix and that $\alpha_{i}=\sum_{j=1}^{m} \gamma_{i, j} \beta_{j}$ for every $i=1, \ldots, m$. By Birkhoff's theorem [11] the doubly stochastic matrix $\left(\gamma_{i, j}\right)$ can be written as a convex combination of permutation matrices, i.e. $\left(\gamma_{i, j}\right)=\sum_{\sigma \in \mathbb{S}_{m}} \eta_{\sigma} P_{\sigma}$, where $\eta_{\sigma} \geq 0, \sum_{\sigma \in \mathbb{S}_{m}} \eta_{\sigma}=1$ and $P_{\sigma}$ is the $m \times m$ permutation matrix induced by $\sigma \in \mathbb{S}_{m}$. Then we have

$$
\alpha_{i}=\sum_{j=1}^{m} \gamma_{i, j} \beta_{j}=(\gamma \cdot \beta)_{i}=\left(\sum_{\sigma \in \mathbb{S}_{m}} \eta_{\sigma} P_{\sigma} \cdot \beta\right)_{i}=\sum_{\sigma \in \mathbb{S}_{m}} \eta_{\sigma} \beta_{\sigma(i)}, \quad 1 \leq i \leq m .
$$

The fact that $\mathcal{M}$ is a $\mathrm{II}_{1}$ factor and that the elements of the partitions $\left\{p_{i}\right\}_{i},\left\{q_{i}\right\}_{i}$ have the same trace guarantees the existence of unitaries $u_{\sigma}$ such that $u_{\sigma} q_{\sigma(i)} u_{\sigma}^{*}=$ $p_{i}, 1 \leq i \leq m$, for every $\sigma \in \mathbb{S}_{m}$. Indeed, if $\sigma \in \mathbb{S}_{m}$, the equalities, $\tau\left(q_{\sigma(i)}\right)=$ $\tau\left(p_{i}\right)$ imply that there exist partial isometries $v_{i, \sigma} \in \mathcal{M}$ such that $v_{i, \sigma} v_{i, \sigma}^{*}=p_{i}$ and $v_{i, \sigma}^{*} v_{i, \sigma}=q_{\sigma(i)}$ for $i=1, \ldots, m$. Then $u_{\sigma}=\sum_{i=1}^{m} v_{i, \sigma} \in \mathcal{M}$ are the required unitaries. Using equation (4), and letting $\rho(\cdot)=\sum_{\sigma \in \mathbb{S}_{m}} \eta_{\sigma} u_{\sigma}(\cdot) u_{\sigma}^{*} \in \mathcal{D}(\mathcal{M})$,

$$
\begin{aligned}
\sum_{i=1}^{m} \alpha_{i} p_{i} & =\sum_{i=1}^{m}\left(\sum_{\sigma \in \mathbb{S}_{m}} \eta_{\sigma} \beta_{\sigma(i)}\right) p_{i}=\sum_{\sigma \in \mathbb{S}_{m}} \eta_{\sigma}\left(\sum_{i=1}^{m} \beta_{\sigma(i)} u_{\sigma} q_{\sigma(i)} u_{\sigma}^{*}\right) \\
& =\sum_{\sigma \in \mathbb{S}_{m}} \eta_{\sigma} u_{\sigma}\left(\sum_{i=1}^{m} \beta_{i} q_{i}\right) u_{\sigma}^{*}=\rho\left(\sum_{i=1}^{m} \beta_{i} q_{i}\right) .
\end{aligned}
$$

Lemma 3.6. Let $\mathcal{B} \subset \mathcal{M}$ be a separable $C^{*}$-subalgebra, and let $\left\{p_{i}\right\}_{i=1}^{m} \subseteq \mathcal{B}^{\prime} \cap \mathcal{M}$ be a partition of the unity. Then there exists a sequence $\left\{\rho_{i}\right\}_{i \in \mathbb{N}} \subset \mathcal{D}(\mathcal{M})$ such that for every $b \in \mathcal{B}$, if we let $\beta_{i}(b)=\tau\left(b p_{i}\right) / \tau\left(p_{i}\right)$, then

$$
\lim _{j \rightarrow \infty}\left\|\rho_{j}(b)-\sum_{i=1}^{m} \beta_{i}(b) p_{i}\right\|=0 \text {. }
$$

Proof. (see section 6).

Theorem 3.7. Let $\mathcal{A}, \mathcal{B} \subseteq \mathcal{M}$ be separable abelian $C^{*}$-subalgebras and let $T \in$ $D S(\mathcal{M})$. Let $\mathcal{S}$ be the operator subsystem of $\mathcal{B}$ given by $\mathcal{S}=T^{-1}(\mathcal{A}) \cap \mathcal{B}$. Then there exists a sequence $\left(\rho_{r}\right)_{r \in \mathbb{N}} \subseteq \mathcal{D}(\mathcal{M})$ such that $\lim _{r \rightarrow \infty}\left\|T(b)-\rho_{r}(b)\right\|=0$ for every $b \in \mathcal{S}$.

Proof. First, note that we just have to prove the theorem for separable diffuse abelian $C^{*}$-subalgebras of $\mathcal{M}$; indeed, assume it holds for such algebras and let $\mathcal{A}, \mathcal{B} \subseteq \mathcal{M}$ be arbitrary separable abelian $C^{*}$-subalgebras. Then, by Theorem 3.2 there exist separable diffuse abelian subalgebras $\tilde{\mathcal{A}}$ and $\tilde{\mathcal{B}}$ of $\mathcal{M}$ such that $\mathcal{A} \subseteq \tilde{\mathcal{A}}$ and $\mathcal{B} \subseteq \tilde{\mathcal{B}}$. 
Thus we get a sequence $\left\{\rho_{r}\right\}_{r \in \mathbb{N}} \subseteq \mathcal{D}$ such that $\lim _{r \rightarrow \infty}\left\|T(b)-\rho_{r}(b)\right\|=0$, for every $b \in T^{-1}(\mathcal{A}) \cap \mathcal{B} \subseteq T^{-1}(\tilde{\mathcal{A}}) \cap \tilde{\mathcal{B}}$. So we assume that $\mathcal{A}$ and $\mathcal{B}$ are diffuse.

By Proposition 3.3, there exists an unbounded set $\mathbb{M} \subseteq \mathbb{N}$ and, for each $m \in \mathbb{M}$, $k(m)$ partitions of the unity $\left\{q_{i}^{j, m}\right\}_{i=1}^{m} \subseteq \mathcal{B}^{\prime} \cap \mathcal{M}$ and $\left\{p_{i}^{j, m}\right\}_{i=1}^{m} \subseteq \mathcal{A}^{\prime} \cap \mathcal{M}$ (in order to simplify the notation we avoid the supra-index $m$ and write $\left.q_{i}^{j}, p_{i}^{j}\right), 1 \leq j \leq k$, such that for every $b \in T(\mathcal{A})^{-1} \cap \mathcal{B}$ and every $r \in \mathbb{N}$, there exists $m_{0}(r, b) \in \mathbb{M}$ such that if $m \geq m_{0}$ we have

$$
\left\|b-\frac{1}{k} \sum_{j=1}^{k}\left(\sum_{i=1}^{m} \beta_{i}^{j} q_{i}^{j}\right)\right\|<\frac{1}{r}
$$

and

$$
\left\|T(b)-\frac{1}{k} \sum_{j=1}^{k}\left(\sum_{i=1}^{m} \alpha_{i}^{j} p_{i}^{j}\right)\right\|<\frac{1}{r}
$$

where $\beta_{i}^{j}=m \tau\left(b q_{i}^{j}\right), \alpha_{i}^{j}=m \tau\left(T(b) p_{i}^{j}\right), \tau\left(p_{i}^{j}\right)=\tau\left(q_{i}^{j}\right)=1 / m$, (from the construction of such partitions it is evident that we can assume that both have the same unbounded set $\mathbb{M}$ and the same $k(m)$ for every $m \in \mathbb{M})$. Fix $b \in \mathcal{B}$. Since $\|T\|=1$, it follows from (5) that

$$
\left\|T(b)-\frac{1}{k} \sum_{j=1}^{k}\left(\sum_{i=1}^{m} \beta_{i}^{j} T\left(q_{i}^{j}\right)\right)\right\| \leq \frac{1}{r} .
$$

Applying to (7) the fact that the linear map in Remark 3.4 is linear and contractive (with $\left\{p_{i}^{j}\right\}_{i}$ as the partitions of the unity), we get

$$
\left\|\frac{1}{k} \sum_{j=1}^{k} \sum_{i=1}^{m} \alpha_{i}^{j} p_{i}^{j}-\frac{1}{k^{2}} \sum_{j=1}^{k}\left(\sum_{t=1}^{k} \sum_{i=1}^{m} \alpha_{i}^{j, t} p_{i}^{t}\right)\right\| \leq \frac{1}{r},
$$

where $\alpha_{i}^{j, t}=m \sum_{l=1}^{m} \beta_{l}^{j} \tau\left(T\left(q_{l}^{j}\right) p_{i}^{t}\right)$, and $\alpha_{i}^{j}$ as defined above. By Lemma 3.5 there exists $\rho_{j, t}^{m} \in \mathcal{D}(\mathcal{M})$ such that

$$
\sum_{i=1}^{m} \alpha_{i}^{j, t} p_{i}^{t}=\rho_{j, t}^{m}\left(\sum_{l=1}^{m} \beta_{l}^{j} q_{l}^{j}\right), \quad 1 \leq j, t \leq k .
$$

Using (6), (8), and (9) we get

$$
\left\|T(b)-\frac{1}{k^{2}} \sum_{j=1}^{k} \sum_{t=1}^{k} \rho_{j, t}^{m}\left(\sum_{l=1}^{m} \beta_{l}^{j} q_{l}^{j}\right)\right\| \leq \frac{2}{r},
$$

By Lemma 3.6 there exist sequences $\left(\tilde{\rho}_{n}^{j}\right)_{n \in \mathbb{N}} \subseteq \mathcal{D}(\mathcal{M}), 1 \leq j \leq k$, independent of $b$, such that for every $r \in \mathbb{N}$ there exists $n_{0}=n_{0}(r, b)$ such that if $n \geq n_{0}$ then

$$
\left\|\sum_{l=1}^{m} \beta_{l}^{j} q_{l}^{j}-\tilde{\rho}_{n}^{j}(b)\right\| \leq \frac{1}{r}, \quad 1 \leq j \leq k .
$$


From (10) and (11), together with the fact that each $\rho \in \mathcal{D}(\mathcal{M})$ is contractive we get, for every $n \geq n_{0}(r, b)$

$$
\left\|T(b)-\frac{1}{k^{2}} \sum_{j=1}^{k} \sum_{t=1}^{k} \rho_{j, t}^{m}\left(\tilde{\rho}_{n}^{j}(b)\right)\right\| \leq \frac{3}{r},
$$

Consider a dense countable subset $\left\{b_{1}, b_{2}, \ldots\right\}$ of $\mathcal{B}$. Now define $n(r), m(r)$ as

$$
n(r)=\max \left\{n_{0}\left(r, b_{1}\right), \ldots, n_{0}\left(r, b_{r}\right)\right\}, \quad m(r)=\max \left\{m_{0}\left(r, b_{1}\right), \ldots, m_{0}\left(r, b_{r}\right)\right\}
$$

and let $\rho_{r}:=\frac{1}{k^{2}} \sum_{j=1}^{k} \sum_{t=1}^{k} \rho_{j, t}^{m(r)} \circ \tilde{\rho}_{n(r)}^{j} \in \mathcal{D}(\mathcal{M})$, where $k=k(m(r))$. Then, from the previous calculations, we see that $\left\|T\left(b_{j}\right)-\rho_{r}\left(b_{j}\right)\right\|<3 / r$ whenever $1 \leq j \leq r$. Let $b \in \mathcal{B}$, and $\epsilon>0$. Then there exists $l \in \mathbb{N}$ such that $\left\|b-b_{l}\right\|<\epsilon / 3$. If $r>\max \{l, 9 / \epsilon\}$, then $\left\|T\left(b_{l}\right)-\rho_{r}\left(b_{l}\right)\right\|<\epsilon / 3$, and so $\left\|T(b)-\rho_{r}(b)\right\| \leq \epsilon$.

Corollary 3.8. Let $T \in D S(\mathcal{M})$ and let $\left(a_{i}\right)_{i=1}^{n},\left(b_{i}\right)_{i=1}^{n} \subseteq \mathcal{M}_{s a}$ be abelian families such that $T\left(b_{i}\right)=a_{i}, 1 \leq i \leq n$. Then there exists a sequence $\left(\rho_{r}\right)_{r \in \mathbb{N}} \subseteq \mathcal{D}(\mathcal{M})$ such that for $1 \leq i \leq n \lim _{r \rightarrow \infty}\left\|a_{i}-\rho_{r}\left(b_{i}\right)\right\|=0$.

Proof. Consider $\mathcal{A}=C^{*}\left(a_{1}, \ldots, a_{n}\right)$ and $\mathcal{B}=C^{*}\left(b_{1}, \ldots, b_{n}\right)$, which are separable abelian $C^{*}$-subalgebras of $\mathcal{M}$. Applying Theorem 3.7 to these algebras we get a sequence $\left(\rho_{r}\right)_{r \in \mathbb{N}} \subseteq \mathcal{D}(\mathcal{M})$ such that $\lim _{r \rightarrow \infty}\left\|T(b)-\rho_{r}(b)\right\|=0$ for every $b \in$ $T^{-1}(\mathcal{A}) \cap \mathcal{B}$. By our choice, $b_{i} \in T^{-1}(\mathcal{A}) \cap \mathcal{B}$ and so $\left\|T\left(b_{i}\right)-\rho_{r}\left(b_{i}\right)\right\|=\left\|a_{i}-\rho_{r}\left(b_{i}\right)\right\| \stackrel{r}{\rightarrow}$ 0 .

\section{DOUbly STOCHASTIC KERNELS AND JOINT MAJORIZATION}

We begin by introducing doubly stochastic kernels, which are a natural generalization of doubly stochastic matrices (see Example 4.2). We shall use them to define joint majorization in analogy with $[22]$.

Definition 4.1. Let $\left(X, \mu_{X}\right),\left(Y, \mu_{Y}\right)$ be two probability spaces. A positive unital linear map $\nu: L^{\infty}\left(Y, \mu_{Y}\right) \rightarrow L^{\infty}\left(X, \mu_{X}\right)$ is said to be a doubly stochastic kernel if $\int_{X} \nu\left(1_{\Delta}\right) d \mu_{X}=\mu_{Y}(\Delta)$, for every $\mu_{Y}$-measurable set $\Delta \subseteq Y$.

Doubly stochastic kernels between probability spaces are norm continuous and normal.

Example 4.2. Let $X$ and $Y$ be compact spaces and let $\mu_{X}$ and $\mu_{Y}$ be regular Borel probability measures in $X$ and $Y$ respectively. Consider $D \in L^{1}\left(\mu_{X} \times \mu_{Y}\right)$ and let $\nu(f)(x)=\int_{Y} D(x, y) f(y) d \mu_{Y}(y)$. Then $\nu: L^{\infty}\left(X, \mu_{X}\right) \rightarrow L^{\infty}\left(Y, \mu_{Y}\right)$ is a doubly stochastic kernel if and only if $D(x, y) \geq 0\left(\mu_{X} \times \mu_{Y}\right)$-a.e. and $\int_{X} D(x, y) d \mu_{X}(x)=1$ $\mu_{Y}$-a.e, $\int_{Y} D(x, y) d \mu_{Y}(y)=1 \mu_{X}$-a.e. In particular, if $\mu_{X}=\mu_{Y}$ is a measure with finite support $\left\{x_{i}\right\}_{i=1}^{m}$ and such that $\mu_{X}\left(\left\{x_{i}\right\}\right)=\frac{1}{m}$ for $1 \leq i \leq m$ then $D$ is a doubly stochastic kernel if and only if the matrix $\left(D\left(x_{i}, x_{j}\right)\right)_{i, j}$ is an $m \times m$ doubly stochastic matrix.

Proposition 4.3. Let $\bar{a}=\left(a_{i}\right)_{i=1}^{n}, \bar{b}=\left(b_{i}\right)_{i=1}^{n} \subseteq \mathcal{M}_{s a}$ be abelian families. Then the following statements are equivalent:

(1) There exists $T \in D S(\mathcal{M})$ such that $T\left(b_{i}\right)=a_{i}, 1 \leq i \leq n$.

(2) There exists a doubly stochastic kernel $\nu: L^{\infty}\left(\sigma(\bar{b}), \mu_{\bar{b}}\right) \rightarrow L^{\infty}\left(\sigma(\bar{a}), \mu_{\bar{a}}\right)$ such that $\nu\left(\pi_{i}\right)=\pi_{i}, 1 \leq i \leq n$. 
Proof. Assume that $T\left(b_{i}\right)=a_{i}, 1 \leq i \leq n$, with $T \in D S(\mathcal{M})$. Let $\mathcal{A}=C^{*}\left(a_{1}, \ldots, a_{n}\right)$ and $\mathcal{B}=C^{*}\left(b_{1}, \ldots, b_{n}\right)$. As $\mathcal{M}$ is a finite von Neumann algebra, there exists a conditional expectation $\mathcal{P}_{\mathcal{A}}: M \rightarrow L^{\infty}(\mathcal{A})$ that commutes with $\tau$. Then $\nu=$ $\Lambda_{\bar{a}}^{-1} \circ \mathcal{P}_{\mathcal{A}} \circ T \circ \Lambda_{\bar{b}}$ is the desired doubly stochastic kernel. Conversely, let us assume the existence of $\nu$ as in $(2)$. Let $\mathcal{P}_{\mathcal{B}}: \mathcal{M} \rightarrow L^{\infty}(\mathcal{B})$ be the conditional expectation onto $L^{\infty}(\mathcal{B})$ that commutes with $\tau$. Define $T=\Lambda_{\bar{a}} \circ \nu \circ \Lambda_{\bar{b}}^{-1} \circ \mathcal{P}_{\mathcal{B}} \in D S(\mathcal{M})$. Clearly $T\left(b_{i}\right)=a_{i}, 1 \leq i \leq n$.

Definition 4.4. Let $\bar{a}=\left(a_{i}\right)_{i=1}^{n}, \bar{b}=\left(b_{i}\right)_{i=1}^{n}$ be two abelian families in $\mathcal{M}_{s a}$. We say that $\bar{a}$ is jointly majorized by $\bar{b}$ (and we write $\bar{a} \prec \bar{b}$ ) if there exists a doubly stochastic kernel $\nu: L^{\infty}\left(\sigma(\bar{b}), \mu_{\bar{b}}\right) \rightarrow L^{\infty}\left(\sigma(\bar{a}), \mu_{\bar{a}}\right)$ such that $\nu\left(\pi_{i}\right)=\pi_{i}, 1 \leq i \leq n$.

If $\left(x_{1}, \ldots, x_{n}\right)$ is a finite family in $\mathcal{M}$, let $\mathcal{U}_{\mathcal{M}}\left(x_{1}, \ldots, x_{n}\right)$ denote the joint unitary orbit of the family with respect to the unitary group $\mathcal{U}_{\mathcal{M}}$ of $\mathcal{M}$, i.e.

$$
\mathcal{U}_{\mathcal{M}}\left(x_{1}, \ldots, x_{n}\right)=\left\{\left(u^{*} x_{1} u, \ldots, u^{*} x_{n} u\right): u \in \mathcal{U}_{\mathcal{M}}\right\} .
$$

We shall also consider the convex hull of the unitary orbit of a family $\left(x_{i}\right)_{i=1}^{n}$,

$$
\operatorname{conv}\left(\mathcal{U}_{\mathcal{M}}\left(x_{i}\right)_{i=1}^{n}\right)=\left\{\left(\rho\left(x_{i}\right)\right)_{i=1}^{n}, \rho \in \mathcal{D}\right\} .
$$

We denote by $\overline{\operatorname{conv}}\left(\mathcal{U}_{\mathcal{M}}\left(x_{i}\right)_{i=1}^{n}\right), \overline{\operatorname{conv}}^{w}\left(\mathcal{U}_{\mathcal{M}}\left(x_{i}\right)_{i=1}^{n}\right)$ and $\overline{\operatorname{conv}}^{1}\left(\mathcal{U}_{\mathcal{M}}\left(x_{i}\right)_{i=1}^{n}\right)$ the respective closures in the coordinate-wise norm topology, coordinate-wise weak operator topology, and coordinate-wise $L^{1}$ topology.

Theorem 4.5. Let $\bar{a}=\left(a_{i}\right)_{i=1}^{n}, \bar{b}=\left(b_{i}\right)_{i=1}^{n}$ be abelian families in $\mathcal{M}_{s a}$. Then the following statements are equivalent:

(1) $\bar{a} \prec \bar{b}$.

(2) $\bar{a} \in \overline{\operatorname{conv}}\left(\mathcal{U}_{\mathcal{M}}(\bar{b})\right)$.

(3) $\bar{a} \in \overline{\operatorname{conv}}^{1}\left(\mathcal{U}_{\mathcal{M}}(\bar{b})\right)$.

(4) $\bar{a} \in \overline{\mathrm{conv}}^{w}\left(\mathcal{U}_{\mathcal{M}}(\bar{b})\right)$.

(5) $\mu_{\bar{a}} \prec \mu_{\bar{b}}$.

(6) There exists a completely positive map $T \in D S(\mathcal{M})$ such that $a_{i}=T\left(b_{i}\right), 1 \leq$ $i \leq n$.

(7) There exists $T \in D S(\mathcal{M})$ such that $a_{i}=T\left(b_{i}\right), 1 \leq i \leq n$.

(8) $\tau\left(f\left(a_{1}, \ldots, a_{n}\right)\right) \leq \tau\left(f\left(b_{1}, \ldots, b_{n}\right)\right)$ for every continuous convex function $f$ : $\mathbb{R}^{n} \rightarrow \mathbb{R}$

The proof of Theorem 4.5 will be split into several lemmas, and all the pieces will be put together at the end of the section.

Remark 4.6. Let $x \in \mathcal{M}$ be a normal operator. Recall (see the last paragraph of section 2.1) that there is a natural way to identify the usual spectral measure of $x$ with that of the abelian pair $(\operatorname{Re}(x), \operatorname{Im}(x))$. If $T \in D S(\mathcal{M})$, then since $T$ is positive $T(x)=y$ if and only if $T(\operatorname{Re}(x))=\operatorname{Re}(y)$ and $T(\operatorname{Im}(x))=\operatorname{Im}(y)$. Using Theorem 4.5 , we see that if $x, y \in \mathcal{M}$ are normal operators then $x \prec y$ in the sense of [16] if and only if $(\operatorname{Re}(x), \operatorname{Im}(x)) \prec(\operatorname{Re}(y), \operatorname{Im}(y))$ in the sense of Definition 4.4.

Let $\mathcal{P}_{\mathcal{N}}$ denote the trace preserving conditional expectation onto the abelian von Neumann subalgebra $\mathcal{N} \subseteq \mathcal{M}$. Using Theorem 4.5 we can then obtain a generalization of Theorem 7.2 in [10]. 
Corollary 4.7. Let $\mathcal{N} \subseteq \mathcal{M}$ be an abelian von Neumann subalgebra and let $\left(b_{i}\right)_{i=1}^{n} \subseteq$ $\mathcal{M}_{s a}$ be an abelian family. Then $\left(\mathcal{P}_{\mathcal{N}}\left(b_{i}\right)\right)_{i=1}^{n} \prec\left(b_{i}\right)_{i=1}^{n}$.

In the remainder of the section we prove the implications needed to prove Theorem 4.5. The single variable case of the following lemma can be found in [16].

Lemma $4.8\left((4) \Rightarrow(6)\right.$ in Theorem 4.5). Let $\bar{a}=\left(a_{i}\right)_{i=1}^{n}, \bar{b}=\left(b_{i}\right)_{i=1}^{n} \subseteq \mathcal{M}_{s a}$ be abelian families and assume that $\bar{a} \in \overline{\mathrm{conv}}^{w}\left(\mathcal{U}_{\mathcal{M}}(\bar{b})\right)$. Then there exists a completely positive $T \in D S(\mathcal{M})$ such that $a_{i}=T\left(b_{i}\right), 1 \leq i \leq n$.

Proof. Let $\left\{\left(b_{1}^{j}, \ldots, b_{n}^{j}\right)\right\}_{j \in J} \subseteq \operatorname{conv}\left(\mathcal{U}_{\mathcal{M}}\left(b_{1}, \ldots, b_{n}\right)\right)$ such that $b_{i}^{j} \underset{j}{\stackrel{\text { weakly }}{\longrightarrow}} a_{i}, 1 \leq i \leq$ $n$. Then there exists a sequence $\left(\rho_{j}\right)_{j \in J} \subseteq \mathcal{D}(\mathcal{M})$ such that $\left(b_{1}^{j}, \ldots, b_{n}^{j}\right)=\left(\rho_{j}\left(b_{1}\right)\right.$, $\ldots, \rho_{j}\left(b_{n}\right)$ ), for every $j \in J$. Note that each $\rho_{j}$ is a completely positive doubly stochastic map. Since the net $\left\{\rho_{j}\right\}_{j \in J}$ is norm bounded, it has an accumulation point in the BW topology [9], i.e. there exists a subnet (which we still call $\left\{\rho_{j}\right\}_{j \in J}$ ) and a completely positive map $T: \mathcal{M} \rightarrow \mathcal{M}$ such that $\rho_{j}(x) \stackrel{\text { weakly }}{\longrightarrow} T(x)$ if $x \in \mathcal{M}$. By normality of the trace, $T$ is trace preserving, positive and unital. Since $\rho_{j}\left(b_{i}\right)=$ $b_{i}^{j} \stackrel{\text { weakly }}{\longrightarrow} a_{i}$, we have $T\left(b_{i}\right)=a_{i}, 1 \leq i \leq n$.

Lemma $4.9\left((1) \Rightarrow(5)\right.$ in Theorem 4.5). Let $\bar{a}=\left(a_{i}\right)_{i=1}^{n}, \bar{b}=\left(b_{i}\right)_{i=1}^{n} \subseteq \mathcal{M}_{s a}$ be abelian families. If $\bar{a} \prec \bar{b}$, then $\mu_{\bar{a}} \prec \mu_{\bar{b}}$.

Proof. By hypothesis $\bar{a} \prec \bar{b}$, so there exists a doubly stochastic kernel $\nu: L^{\infty}\left(\sigma(\bar{b}), \mu_{\bar{b}}\right) \rightarrow$ $L^{\infty}\left(\sigma(\bar{a}), \mu_{\bar{a}}\right)$ such that $\nu\left(\pi_{i}\right)=\pi_{i}, 1 \leq i \leq n$. Let $\nu_{1}, \ldots, \nu_{m} \in M_{+}^{\sim}\left(\mathbb{R}^{n}\right)$ with $\sum_{j=1}^{m} \nu_{j}=\mu_{\bar{a}}$. Define measures $\nu_{j}^{\prime}$ by $\nu_{j}^{\prime}(\Delta)=\nu_{j}\left(\nu\left(1_{\Delta}\right)\right)$. By continuity of $\nu$, $\nu_{j}^{\prime}(f)=\nu_{j}(\nu(f))$ for every $f \in L^{\infty}\left(\sigma(\bar{b}), \mu_{\bar{b}}\right)$. So $\nu_{j}^{\prime}\left(\pi_{i}\right)=\nu_{j}\left(\nu\left(\pi_{i}\right)\right)=\nu_{j}\left(\pi_{i}\right)$, $1 \leq i \leq n$ and $1 \leq j \leq m$, and similarly $\nu_{j}(1)=\nu_{j}^{\prime}(1)$, so that $\nu_{j} \sim \nu_{j}^{\prime}$, for $1 \leq j \leq m$. Finally, $\sum_{j=1}^{m} \nu_{j}^{\prime}(\Delta)=\sum_{j=1}^{m} \nu_{j}\left(\nu\left(1_{\Delta}\right)\right)=\mu_{\bar{a}}\left(\nu\left(1_{\Delta}\right)\right)=\mu_{\bar{b}}(\Delta)$. So $\sum_{j=1}^{m} \nu_{j}^{\prime}=\mu_{\bar{b}}$. We conclude that $\mu_{\bar{a}} \prec \mu_{\bar{b}}$.

Lemma $4.10\left((5) \Rightarrow(1)\right.$ in Theorem 4.5). Let $\bar{a}=\left(a_{i}\right)_{i=1}^{n}, \bar{b}=\left(b_{i}\right)_{i=1}^{n} \subset \mathcal{M}_{s a}$ be abelian families. If $\mu_{\bar{a}} \prec \mu_{\bar{b}}$, then there exists $T \in D S(\mathcal{M})$ such that $T\left(b_{i}\right)=a_{i}$, $1 \leq i \leq n$.

Proof. By compactness, we can consider partitions $\left\{\Delta_{j}^{r}\right\}_{j=1}^{m(r)}$ of $\sigma(\bar{a})$ with $\operatorname{diam}\left(\Delta_{j}^{r}\right)<$ $1 / r$ for every $1 \leq j \leq m$. Fix points $x_{1}^{r}, \ldots, x_{m(r)}^{r}$ with $x_{j}^{r} \in \Delta_{j}^{r}$ and define measures $\mu_{j}^{r}$ by $\mu_{j}^{r}(\cdot)=\mu_{\bar{a}}\left(\cdot \cap \Delta_{j}^{r}\right)$. Then clearly $\sum_{j} \mu_{j}^{r}=\mu_{\bar{a}}$. As $\mu_{\bar{a}} \prec \mu_{\bar{b}}$ by hypothesis, there exist measures $\nu_{j}^{r}$ with $\nu_{j}^{r} \sim \mu_{j}^{r}$ and $\sum_{j} \nu_{j}^{r}=\mu_{\bar{b}}$. Let $g_{j}^{r}$ be the Radon-Nikodym derivatives $g_{j}^{r}=d \nu_{j}^{r} / d \mu_{\bar{b}}$. Note that $\sum_{j} g_{j}^{r}=1$ ( $\mu_{\bar{b}}-$ a.e. $)$. Define a function $D_{r}: \sigma(\bar{a}) \times \sigma(\bar{b}) \rightarrow \mathbb{R}$ by

$$
D_{r}(s, t)=\sum_{j=1}^{m(r)} \frac{g_{j}^{r}(t)}{\mu_{a}\left(\Delta_{j}^{r}\right)} 1_{\Delta_{j}^{r}}(s) .
$$


We will use the kernels $D_{r}$ to approximate $T$. Let us define $\nu_{r}: L^{\infty}\left(\sigma(\bar{b}), \mu_{\bar{b}}\right) \rightarrow$ $L^{\infty}\left(\sigma(\bar{a}), \mu_{\bar{a}}\right)$ by

$$
\nu_{r}(b)(s)=\int_{\sigma(\bar{b})} b(t) D_{r}(s, t) d \mu_{\bar{b}}(t) .
$$

The map $\nu_{r}$ can be seen to be doubly stochastic using the equivalence $\mu_{j}^{r} \sim \nu_{j}^{r}$. By Proposition 4.3 there is an associated sequence $\left\{T_{r}\right\}_{r} \subset D S(\mathcal{M})$ such that $T_{r}\left(b_{i}\right)=$ $\int_{\sigma(\bar{a})} \nu_{r}\left(\pi_{i}\right) d E_{\bar{a}} \in L^{\infty}(\mathcal{A}), 1 \leq i \leq n$. The bounded net $\left\{T_{r}\right\}_{r \in \mathbb{N}}$ has a subnet $\left\{T_{k}\right\}_{k \in K}$ that converges to a cluster point $T \in D S(\mathcal{M})$ in the BW topology. Since this subnet is bounded, $T\left(b_{i}\right)=w-\lim _{k \in K} T_{k}\left(b_{i}\right) \in L^{\infty}(\mathcal{A})$. We claim that $T\left(b_{i}\right)=$ $a_{i}, 1 \leq i \leq n$. To see this, since the net $\left\{T_{k}\left(b_{i}\right)\right\}_{k \in K}$ is bounded, we just have to prove that

$$
\lim _{k} \tau\left(x T_{k}\left(b_{i}\right)\right)=\tau\left(x a_{i}\right), \quad 1 \leq i \leq n, \quad \forall x \in \mathcal{A} .
$$

Equivalently, we have to show that for every continuous function $f \in C(\sigma(\bar{a}))$ and every $i=1, \ldots, n$,

$$
\lim _{k} \int_{\sigma(\bar{a})} f(s)\left(\int_{\sigma(\bar{b})} D_{k}(s, t) \pi_{i}(t) d \mu_{\bar{b}}(t)\right) d \mu_{\bar{a}}(s)=\int_{\sigma(\bar{a})} f(s) \pi_{i}(s) d \mu_{\bar{a}}(s) .
$$

This can be seen by a standard approximation argument, using the uniform continuity of $f$, the fact that the diameters of $\Delta_{j}^{r}$ tend to 0 as $r$ increases, and the equivalence $\mu_{j}^{r} \sim \nu_{j}^{r}$.

Proof of Theorem 4.5. Proposition 4.3 shows the equivalence $(7) \Leftrightarrow(1)$ and Corollary 3.8 is $(7) \Rightarrow(2)$. The implication $(2) \Rightarrow(3) \Rightarrow(4)$ is trivial. Lemma 4.8 shows that $(4) \Rightarrow(6)$, and it is clear that $(6) \Rightarrow(7)$. Lemmas $4.9,4.10$ and Proposition 4.3 prove the equivalence $(5) \Leftrightarrow(1)$. So we have that (1)-(7) are equivalent. Finally, Corollary 2.3 shows that $(5) \Leftrightarrow(8)$.

\section{Joint unitary orbits of abelian families in $\mathcal{M}_{s a}$}

Given families $\bar{a}=\left(a_{i}\right)_{i=1}^{n}, \bar{b}=\left(b_{i}\right)_{i=1}^{n} \subseteq \mathcal{M}$, we say that $\bar{a}$ and $\bar{b}$ are jointly approximately unitarily equivalent in $\mathcal{M}$ if $\bar{a} \in \overline{\mathcal{U}_{\mathcal{M}}(\bar{b})}$, that is if there exists a sequence of unitary operators $\left(u_{n}\right)_{n \in \mathbb{N}} \subseteq \mathcal{M}$ such that $\lim _{n \rightarrow \infty}\left\|u_{n} b_{i} u_{n}^{*}-a_{i}\right\|=0$ for every $i=1 \ldots, n$. It is clear that this is an equivalence relation. Moreover, if $\bar{a}$ and $\bar{b}$ are jointly approximately unitarily equivalent in $\mathcal{M}$ then $\bar{a}$ is an abelian family if and only if $\bar{b}$ is. In [10] a characterization of approximately unitarily equivalence between selfadjoint operators is obtained in terms of the spectral distributions. The main result of this section, Theorem 5.1, characterizes this relation for abelian families in $\mathcal{M}_{s a}$.

Theorem 5.1. Let $\bar{a}=\left(a_{i}\right)_{i=1}^{n}$ and $\bar{b}=\left(b_{i}\right)_{i=1}^{n} \subset \mathcal{M}_{s a}$ be abelian families. Then the following statements are equivalent:

(1) $\bar{a}$ and $\bar{b}$ are jointly approximately unitary equivalent in $\mathcal{M}$.

(2) $\bar{a} \prec \bar{b}$ and $\bar{b} \prec \bar{a}$

(3) $\mu_{\bar{a}}=\mu_{\bar{b}}$

(4) $\tau\left(f\left(a_{1}, \ldots, a_{n}\right)\right)=\tau\left(f\left(b_{1}, \ldots, b_{n}\right)\right)$ for every continuous convex function $f$ : $\mathbb{R}^{n} \rightarrow \mathbb{R}$. 
(5) $\tau\left(f\left(a_{1}, \ldots, a_{n}\right)\right)=\tau\left(f\left(b_{1}, \ldots, b_{n}\right)\right)$ for every continuous function $f: \mathbb{R}^{n} \rightarrow$ $\mathbb{R}$.

Proof. By Theorem 4.5 we have $(1) \Rightarrow(2)$ and $(2) \Leftrightarrow(4)$. Moreover, (4) is equivalent to $\mu_{\bar{a}}(f)=\mu_{\bar{b}}(f)$ for every convex function $f$. Then $\mu_{\bar{a}}(f)=\mu_{\bar{b}}(f)$ for every continuous function $f$ [3, Proposition I.1.1], and so $\mu_{\bar{a}}=\mu_{\bar{b}}$. Therefore (4) $\Rightarrow(5) \Rightarrow(3)$. Again, by Theorem $4.5(3) \Rightarrow(2)$ and so $(2)-(5)$ are equivalent. Finally, we prove that $(3) \Rightarrow(1)$. If we assume that $\mu_{\bar{a}}=\mu_{\bar{b}}$ then $\sigma(\bar{a})=\operatorname{supp} \mu_{\bar{a}}=\operatorname{supp} \mu_{\bar{b}}=\sigma(\bar{b})$ and for every Borel set $\Delta$ in $\sigma(\bar{a})$ we have

$$
\tau\left(E_{\bar{a}}(\Delta)\right)=\mu_{\bar{a}}\left(1_{\Delta}\right)=\mu_{\bar{b}}\left(1_{\Delta}\right)=\tau\left(E_{\bar{b}}(\Delta)\right) .
$$

Let $\epsilon>0$. By compactness, choose $B_{1}, \ldots, B_{m}$ to be a finite disjoint covering of $\sigma(\bar{a})=\sigma(\bar{b})$ such that there are points $x_{j} \in B_{j}$ with the property that $\mid \pi_{i}(\lambda)-$ $\pi_{i}\left(x_{j}\right) \mid<\epsilon / 2$ for every $\lambda \in B_{j}, 1 \leq i \leq n, 1 \leq j \leq m$. Then we get, using the Spectral Theorem,

$$
\left\|a_{i}-\sum_{j=1}^{m} \pi_{i}\left(x_{j}\right) E_{\bar{a}}\left(B_{j}\right)\right\|<\frac{\epsilon}{2},\left\|b_{i}-\sum_{j=1}^{m} \pi_{i}\left(x_{j}\right) E_{\bar{b}}\left(B_{j}\right)\right\|<\frac{\epsilon}{2}
$$

for $i=1, \ldots, n$. From equation (13) we get that $\tau\left(E_{\bar{a}}\left(B_{j}\right)\right)=\tau\left(E_{\bar{b}}\left(B_{j}\right)\right)$ for every $j=1, \ldots, m$. As in the proof of Lemma 3.5, we get a unitary $w_{\epsilon} \in \mathcal{U}(\mathcal{M})$ such that $w_{\epsilon}^{*} E_{\bar{b}}\left(B_{j}\right) w_{\epsilon}=E_{\bar{a}}\left(B_{j}\right)$ for every $j$. Then

$$
w_{\epsilon}^{*}\left(\sum_{j=1}^{m} \pi_{i}\left(x_{j}\right) E_{\bar{b}}\left(B_{j}\right)\right) w_{\epsilon}=\sum_{j=1}^{m} \pi_{i}\left(x_{j}\right) E_{\bar{a}}\left(B_{j}\right) .
$$

Finally, for every $i$ we have

$$
\left\|w_{\epsilon}^{*} b_{i} w_{\epsilon}-a_{i}\right\| \leq\left\|w_{\epsilon}^{*}\left(b_{i}-\sum_{j=1}^{m} \pi_{i}\left(x_{j}\right) E_{\bar{b}}\left(B_{j}\right)\right) w_{\epsilon}\right\|+\frac{\epsilon}{2}<\epsilon .
$$

Corollary 5.2. Let $\Theta$ be a *-automorphism of $\mathcal{M}$. Then $\left.\Theta\right|_{\mathcal{A}}$ is approximately inner for each separable abelian $C^{*}$ subalgebra $\mathcal{A} \subset \mathcal{M}$.

Proof. The uniqueness of the trace guarantees that $\Theta$ is trace-preserving. Being multiplicative, the range of an abelian set will be again abelian. So $\Theta$ is a DS map that takes an abelian family in $\mathcal{M}$ into another. Consider a countable dense subset $\left\{a_{i}\right\}$ of $\mathcal{A}$, and use Theorem 5.1 to obtain unitaries $u_{n}$ for each finite subset $\left\{a_{1}, \ldots, a_{n}\right\}$. An $\epsilon / 3$ argument shows then that the sequence $\left\{\operatorname{Ad} u_{n}\right\}$ approximates $\Theta$ in all of $\mathcal{A}$.

Given $\bar{x}=\left(x_{i}\right)_{i=1}^{n} \subseteq \mathcal{M}$ we denote by $\overline{\mathcal{U}} \mathcal{M}(\bar{x})^{s}$ the closure in the coordinate-wise strong operator topology. An immediate consequence of Theorem 5.1 is that the norm closure of the unitary orbit of a selfadjoint abelian family in a $\mathrm{II}_{1}$ factor is strongly closed. This generalizes [10, Theorem 5.4] and [26, Theorem 8.12(1)]:

Corollary 5.3. Let $\bar{a}=\left(a_{i}\right)_{i=1}^{n} \subseteq \mathcal{M}_{\text {sa }}$ be an abelian family. Then $\overline{\mathcal{U}}_{\mathcal{M}}(\bar{a})^{\|\|}=$ $\overline{\mathcal{U}}_{\mathcal{M}(\bar{a})}{ }^{s}$. 
Proof. Let $\bar{b}=\left(b_{i}\right)_{i=1}^{n} \in{\overline{\mathcal{U}_{\mathcal{M}}(\bar{a})}}^{s}$. There exists a net $\left(b_{1}^{j}, \ldots, b_{n}^{j}\right)_{j \in J} \subseteq \mathcal{U}_{\mathcal{M}}(\bar{a})$ such that $b_{i}^{j}$ converges strongly to $b_{i}$ for each $i=1, \ldots, n$. Let $f: \mathbb{R}^{n} \rightarrow \mathbb{R}$ be a continuous function. Then $\tau\left(f\left(b_{1}^{j}, \ldots, b_{n}^{j}\right)\right)=\tau\left(f\left(a_{1}, \ldots, a_{n}\right)\right)$ for every $j$. Using [27, Lemma II.4.3] we conclude that $\tau\left(f\left(b_{1}, \ldots, b_{n}\right)\right)=\tau\left(f\left(a_{1}, \ldots, a_{n}\right)\right)$. So (5) of Theorem 5.1 implies that $\bar{b} \in \overline{\mathcal{U}_{\mathcal{M}}(\bar{a})}$. The other inclusion is trivial.

\section{Some technical Results}

In this section we prove several results presented in section 3. First we show that any separable abelian $C^{*}$-subalgebra of $\mathcal{M}$ can be embedded into a separable diffuse abelian $C^{*}$-subalgebra. Then we prove some approximation results that hold for separable diffuse abelian $C^{*}$ subalgebras of $\mathcal{M}$.

6.1. Refinements of spectral measures. We begin by recalling some elementary facts about inclusions of abelian $C^{*}$ algebras. If $\mathcal{A} \subseteq \mathcal{B}$ are unital abelian $C^{*}$ algebras, then the function $\Phi: \Gamma(\mathcal{B}) \rightarrow \Gamma(\mathcal{A})$ given by $\Phi(\gamma)=\left.\gamma\right|_{\mathcal{A}}$ is a continuous surjection onto $\Gamma(\mathcal{A})$. If we assume further that $\mathcal{A} \subseteq \mathcal{B} \subseteq \mathcal{M}$ are separable and that $E_{\mathcal{A}}, E_{\mathcal{B}}$ denote their spectral measures, then $E_{\mathcal{A}}=E_{\mathcal{B}} \circ \Phi^{-1}$ and $\mu_{\mathcal{A}}=\mu_{\mathcal{B}} \circ \Phi^{-1}$.

Note that $\operatorname{At}\left(\mu_{\mathcal{A}}\right)=\operatorname{At}\left(E_{\mathcal{A}}\right)$ where $\operatorname{At}\left(E_{\mathcal{A}}\right)$ is the set of atoms of the spectral measure $E_{\mathcal{A}}$ (see the beginning of section 3). Let $\sum_{x \in \operatorname{At}\left(E_{\mathcal{A}}\right)} \mu_{\mathcal{A}}(\{x\})$ be the total atomic mass of $E_{\mathcal{A}}$. Since $\mu_{\mathcal{A}}$ is finite, the total atomic mass is bounded and thus the set of atoms is a countable set.

The two results below lead to the proof of Theorem 3.2.

Lemma 6.1. With the notations above, if $x \in A t\left(E_{\mathcal{B}}\right)$ then $\Phi(x) \in A t\left(E_{\mathcal{A}}\right)$, and the total atomic mass of $\mathcal{B}$ is smaller that the total atomic mass of $\mathcal{A}$.

Proof. Let $x \in \operatorname{At}\left(E_{\mathcal{B}}\right)$ and note that $0<\mu_{\mathcal{B}}(\{x\}) \leq \mu_{\mathcal{B}}\left(\Phi^{-1}(\Phi(\{x\}))\right)=\mu_{\mathcal{A}}(\Phi(\{x\}))$ so $\Phi(x) \in \operatorname{At}\left(E_{\mathcal{A}}\right)=\operatorname{At}\left(\mu_{\mathcal{A}}\right)$. We consider the equivalence relation in $\operatorname{At}\left(E_{\mathcal{B}}\right)$ induced by $\Phi$, i.e. $x \sim y$ if $\Phi(x)=\Phi(y)$. If $Q \in \mathcal{Q}:=\operatorname{At}\left(E_{\mathcal{B}}\right) / \sim$ is such that $\Phi(x)=x_{Q}$ for every $x \in Q$, then using that $Q$ is countable we get $\sum_{x \in Q} \mu_{\mathcal{B}}(\{x\})=$ $\mu_{\mathcal{B}}(Q) \leq \mu_{\mathcal{B}}\left(\Phi^{-1}\left(\left\{x_{Q}\right\}\right)\right)=\mu_{\mathcal{A}}\left(\left\{x_{Q}\right\}\right)$. Therefore

$$
\sum_{x \in \operatorname{At}\left(E_{\mathcal{B}}\right)} \mu_{\mathcal{B}}(\{x\})=\sum_{Q \in \mathcal{Q}} \sum_{x \in Q} \mu_{\mathcal{B}}(\{x\}) \leq \sum_{Q \in \mathcal{Q}} \mu_{\mathcal{A}}\left(\left\{x_{Q}\right\}\right) \leq \sum_{x \in \operatorname{At}\left(E_{\mathcal{A}}\right)} \mu_{\mathcal{A}}(\{x\}) .
$$

Proposition 6.2. With the notations above, let $x_{0} \in \Gamma(\mathcal{A})$ be an atom of $E_{\mathcal{A}}$ and let $\alpha, \beta \in \mathbb{R}$ with $0<\alpha<\beta$. Then there exists $a \in \mathcal{A}^{\prime} \cap \mathcal{M}_{\text {sa }}$ with $[\alpha, \beta] \subseteq \sigma(a) \subseteq$ $[\alpha, \beta] \cup\{0\}, P_{\overline{R(a)}}=E_{\mathcal{A}}\left(\left\{x_{0}\right\}\right)$, and such that if $\mathcal{B}=C^{*}(\mathcal{A}, a) \subset \mathcal{M}$, then $E_{\mathcal{B}}$ has no atoms in the fibre $\Phi^{-1}\left(x_{0}\right)$.

Proof. Let $p=E_{\mathcal{A}}\left(\left\{x_{0}\right\}\right)$ and consider a masa $\tilde{\mathcal{A}} \subset \mathcal{M}$ such that $\mathcal{A} \subset \tilde{\mathcal{A}}$. Then $p \tilde{\mathcal{A}}$ is a masa in the $\mathrm{II}_{1}$ factor $p \mathcal{M} p$, where the trace is $\tau_{p}=\frac{1}{\tau(p)} \tau$. It is well known that there exists a countably generated, non-atomic von Neumann subalgebra $\tilde{\mathcal{A}}_{0}$ of $p \tilde{\mathcal{A}}$ such that there is a von Neumann algebra isomorphism $\Phi: L^{\infty}([0,1], m) \rightarrow \tilde{\mathcal{A}}_{0}$, with $m$ the Lebesgue measure on $[0,1]$, and $\tau_{p}(\Phi(f))=\int_{0}^{1} f d m$. Put $\tilde{a}=\Phi(i d)$; it is clear that $\tilde{a}$ has no atoms in its spectrum with the exception of 0 , and that $E_{\tilde{a}}(\{0\})=1-p, \sigma(a)=[0,1]$. Let $a=(\beta-\alpha) \tilde{a}+\alpha p$, so $[\alpha, \beta] \subseteq \sigma(a) \subseteq[\alpha, \beta] \cup\{0\}$, 
$P_{\overline{R(a)}}=p=E_{\mathcal{A}}\left(\left\{x_{0}\right\}\right)$. As $p$ is a minimal projection in $L^{\infty}(\mathcal{A})$, for every $b \in \mathcal{A}$ we have $p b=p b p=\lambda_{b} p$ and so $a b=a p b=\lambda_{b} p a=b p a=b a$. Thus $a \in \mathcal{A}^{\prime} \cap \mathcal{M}$.

Let $\mathcal{B}=C^{*}(\mathcal{A}, a)$ and let $\Phi: \Gamma(\mathcal{B}) \rightarrow \Gamma(\mathcal{A}), \Psi: \Gamma(\mathcal{B}) \rightarrow \Gamma\left(C^{*}(a)\right)$ be the continuous surjections induced by the inclusions $\mathcal{A} \subseteq \mathcal{B}$ and $C^{*}(a) \subseteq \mathcal{B}$. Note that the restriction $\left.\Psi\right|_{\Phi^{-1}\left(x_{0}\right)}$ is injective. Indeed, let $x, y \in \Phi^{-1}\left(x_{0}\right)$ be such that $\Psi(x)=\Psi(y)$, i.e. the restriction of the characters to $C^{*}(a)$ coincide. Since $\Phi(x)=$ $\Phi(y)\left(=x_{0}\right)$, the characters also coincide on $\mathcal{A}$ and therefore are equal as characters in $\mathcal{B}$, since $\mathcal{B}$ is generated by $\mathcal{A}$ and $C^{*}(a)$.

On the other hand, if $x \in \Gamma(\mathcal{B})$ is such that $x(a) \neq 0$, then $\Phi(x)=x_{0}$. Indeed, assume that $\Phi(x) \neq x_{0}$. Let $f \in C(\Gamma(\mathcal{A}))$ with $f(\Phi(x))=0$ and $f\left(x_{0}\right)=1$. So $f \circ \Phi \geq 1_{\Phi^{-1}\left(x_{0}\right)}$. But then

$$
\int_{\Gamma(\mathcal{B})} f \circ \Phi d E_{\mathcal{B}} \geq \int_{\Gamma(\mathcal{B})} 1_{\Phi^{-1}\left(x_{0}\right)} d E_{\mathcal{B}}=E_{\mathcal{B}}\left(\Phi^{-1}\left(x_{0}\right)\right)=E_{\mathcal{A}}\left(\left\{x_{0}\right\}\right)=p .
$$

Note that if $0 \in \sigma(a)$ then it is an isolated point, so in any case we have $p \in C^{*}(a) \subseteq$ $\mathcal{B}$. Then $0=f \circ \Phi(x) \geq x(p) \geq 0$, so $x(p)=0$. Since $0 \leq a \leq \beta p, x(a)=0$ and the claim follows.

Now let $z \in \Phi^{-1}\left(x_{0}\right)$. If $z(a) \neq 0$, from the first part of the proof we deduce that $\Psi^{-1}(\Psi(z))=\{z\}$. Therefore $E_{\mathcal{B}}(\{z\})=E_{\mathcal{A}}(\{\Psi(z\}))=0$, since $\Psi(z)(a) \neq 0$ and $\operatorname{At}\left(E_{\mathcal{A}}\right) \subseteq\{0\}$. If $z(a)=0$, then

$$
\begin{aligned}
\{z\} & =\Phi^{-1}\left(x_{0}\right) \backslash\left\{x \in \Phi^{-1}\left(x_{0}\right): x(a) \neq 0\right\} \\
& =\Phi^{-1}\left(x_{0}\right) \backslash \Psi^{-1}\left(\left\{x \in \Gamma\left(C^{*}(a)\right): x(a) \neq 0\right\}\right)
\end{aligned}
$$

and

$$
\begin{aligned}
E_{\mathcal{B}}\left(\Psi^{-1}\left(\left\{x \in \Gamma\left(C^{*}(a)\right): x(a) \neq 0\right\}\right)\right) & =E_{\mathcal{A}}\left(\left\{x \in \Gamma\left(C^{*}(a)\right): x(a) \neq 0\right\}\right) \\
& =E_{\mathcal{A}}\left(\left\{x_{0}\right\}\right)=E_{\mathcal{B}}\left(\Phi^{-1}\left(x_{0}\right)\right) .
\end{aligned}
$$

From this we conclude that $E_{\mathcal{B}}(\{z\})=0$.

Proof of Theorem 3.2. Recall that the set $\operatorname{At}\left(E_{\mathcal{A}}\right)$ of atoms of $E_{\mathcal{A}}$ is a (possibly infinite) countable set. If $\operatorname{At}\left(E_{\mathcal{A}}\right)=\emptyset$ then $E_{\mathcal{A}}$ is already diffuse and we are done. Otherwise, let us enumerate $\operatorname{At}\left(E_{\mathcal{A}}\right)=\left\{x_{i}: 1 \leq i \leq r\right\}$, where $r \in \mathbb{N} \cup\{\infty\}$. For $1 \leq i \leq r$, let $I_{i}=\left[1+\frac{1}{2 n}, 1+\frac{1}{2 n-1}\right]$. Then $I_{i} \cap \overline{\bigcup_{1 \leq i \neq j \leq r} I_{j}}=\emptyset$ and $\bigcup_{i=1}^{r} I_{i} \subseteq[1,2]$. For each $i=1, \ldots, r$ there exists, by Proposition 6.2, $a_{i} \in \mathcal{A}^{\prime} \cap \mathcal{M}_{s a}$ such that $P_{\overline{R\left(a_{i}\right)}}=E_{\mathcal{A}}\left(\left\{x_{i}\right\}\right), I_{i} \subseteq \sigma\left(a_{i}\right) \subseteq I_{i} \cup\{0\}$, and such that $E_{\mathcal{A}_{i}}$ has no atoms in the fibre $\Phi_{i}^{-1}\left(x_{i}\right)$, where $\Phi_{i}: \Gamma\left(\mathcal{A}_{i}\right) \rightarrow \mathcal{A}$ denotes the continuous surjection induced by the inclusion $\mathcal{A} \subseteq \mathcal{A}_{i}:=C^{*}\left(\mathcal{A}, a_{i}\right)$. Let $a=\sum_{i=1}^{r} a_{i} \in \mathcal{A}^{\prime} \cap \mathcal{M}_{s a}$ (this sum converges strongly because the ranges of the operators $a_{i}$ are orthogonal and $\left\|a_{i}\right\| \leq 2$ for every $i)$. Then $\mathcal{B}=C^{*}(\mathcal{A}, a)$ is an abelian subalgebra of $\mathcal{M}$.

We claim that the spectral measure $E_{\mathcal{B}}$ of $\mathcal{B}$ has no atoms. Indeed, first note that $1_{I_{i}} \in C\left(\cup_{1 \leq j \leq r} I_{j}\right)$ is a continuous function (because the distance between the sets $I_{i}$ and $\cup_{i \neq j} I_{j}$ is positive); then, since $1_{I_{i}}(a)=a_{i}$, it follows that $\mathcal{A}_{i} \subset \mathcal{B}$ for every $i=1, \ldots, r$. Assume now that $x \in \operatorname{At}(\Gamma(\mathcal{B}))$ and let $\Phi: \Gamma(\mathcal{B}) \rightarrow \Gamma(\mathcal{A})$ be as before. By Lemma 6.1 there exists $i \in\{1, \ldots, r\}$ such that $\Phi(x)=x_{i} \in \operatorname{At}\left(E_{\mathcal{A}}\right)$. Since $\Phi=\Phi_{i} \circ \Psi_{i}$, where $\Psi_{i}: \Gamma(\mathcal{B}) \rightarrow \Gamma\left(\mathcal{A}_{i}\right)$ is the surjection induced by the inclusion $\mathcal{A}_{i} \subseteq \mathcal{B}$, we conclude that $\Psi_{i}(x) \in \Phi_{i}^{-1}\left(x_{i}\right)$ is an atom of the measure $E_{\mathcal{A}_{i}}$, again by 
Lemma 6.1. But this last assertion is a contradiction because by construction there are no atoms in the fibre $\Phi_{i}^{-1}\left(x_{i}\right)$.

Remark 6.3. Given an abelian $C^{*}$ subalgebra $\mathcal{A} \subset \mathcal{M}$, a direct way to find an abelian $C^{*}$-subalgebra $\mathcal{A} \subseteq \tilde{\mathcal{A}} \subset \mathcal{M}$ with diffuse spectral measure is to consider a masa in $\mathcal{M}$ that contains $\mathcal{A}$. Theorem 3.2 shows that $\tilde{\mathcal{A}}$ can be chosen separable (as a $C^{*}$-algebra) whenever $\mathcal{A}$ is separable. When this is the case, the character space of $\tilde{\mathcal{A}}$ is metrizable, a fact that is crucial for our calculations.

6.2. Discrete approximations in separable diffuse abelian algebras. Given a compact metric space it is always possible to find, using uniform continuity, discrete uniform approximations of continuous functions by linear combinations of characteristic functions of certain sets $\left\{Q_{i}\right\}_{i=1}^{m}$. But if we consider a measure on this space and we require equal measures for these sets, there might not be any good uniform approximation based on characteristic functions (even for measures of compact support in the real line). Proposition 3.3 is an intermediate solution to this problem. It was inspired by the proof of [16, Lemma 4.1]. The idea is to use convex combinations to "distribute" the part of the projections that does not fit in an equal measure partition.

Proof of Proposition 3.3. The space $\Gamma(\mathcal{B})$ is a metrizable compact topological space, so we consider a metric $d$ in $\Gamma(\mathcal{B})$ inducing its topology. Let $r \in \mathbb{N}$; by compactness, there exists a partition $\left\{\tilde{Q}_{i}\right\}_{i=1}^{k_{0}}$ of $\Gamma(\mathcal{B})$ with $\operatorname{diam}_{d}\left(\tilde{Q}_{i}\right)<\frac{1}{r}$ and $\sum_{i=1}^{k_{0}} \mu_{\mathcal{B}}\left(\tilde{Q}_{i}\right)=1$. Let $m=m(r)$ be such that $1 / m \leq \min \left\{\mu_{\mathcal{B}}\left(\tilde{Q}_{j}\right)^{2}: 1 \leq j \leq k_{0}\right\}$. Then for $1 \leq j \leq k_{0}$ there exists $k_{j} \in \mathbb{N}$ such that $\mu_{\mathcal{B}}\left(\tilde{Q}_{j}\right)=k_{j} / m+\delta_{j}$ with $0 \leq \delta_{j}<1 / m$. If we let $\tilde{k}=\tilde{k}(r)=\min _{j}\left\{k_{j}\right\}$ then $\tilde{k} \geq \max \left\{\mu_{\mathcal{B}}\left(\tilde{Q}_{j}\right)^{-1}, 1 \leq j \leq k_{0}\right\}$.

For $t=1, \ldots, k_{0}$, choose $\tilde{k}$ partitions $\left\{\tilde{Q}_{j, s}^{t}\right\}_{s=0}^{k_{j}}$ of each $\tilde{Q}_{j}(1 \leq t \leq \tilde{k})$, with $\mu_{\mathcal{B}}\left(\tilde{Q}_{j, s}^{t}\right)=1 / m$ if $1 \leq s \leq k_{j}$ and $\mu_{\mathcal{B}}\left(\tilde{Q}_{j, 0}^{t}\right)=\delta_{j}$, in such a way that $\tilde{Q}_{j, 0}^{t} \subset \tilde{Q}_{j, t}^{1}$, $2 \leq t \leq \tilde{k}$. Note that we can always make such a choice: using Lemma 2.4 choose $\tilde{Q}_{j, 0}^{t} \subseteq \tilde{Q}_{j, t}^{1}$ with $\mu_{\mathcal{B}}\left(\tilde{Q}_{j, 0}^{t}\right)=\delta_{j}<1 / m$, and then take a partition $\left\{\tilde{Q}_{j, s}^{t}\right\}_{s=1}^{k_{j}}$ of $\tilde{Q}_{j} \backslash \tilde{Q}_{j, 0}^{t}$ using again Lemma 2.4 (note that $\mu_{\mathcal{B}}\left(\tilde{Q}_{j} \backslash \tilde{Q}_{j, 0}^{t}\right)=k_{j} / m$ ). By this choice, $\tilde{Q}_{j, 0}^{t} \cap \tilde{Q}_{j, 0}^{t^{\prime}}=\emptyset$ if $t \neq t^{\prime}$.

For each $t=1, \ldots, \tilde{k}$, let $\tilde{Q}_{0,0}^{t}=\cup_{j=1}^{k_{0}} \tilde{Q}_{j, 0}^{t}$. Then $\mu_{\mathcal{B}}\left(\tilde{Q}_{0,0}^{t}\right)=1-\sum_{j} k_{j} / m=$ $\left(m-\sum_{j=1}^{k_{0}} k_{j}\right) / m$. Finally, make partitions of each set $\tilde{Q}_{0,0}^{t}$ into $n_{1}=m-\sum_{j} k_{j}$ subsets $\left\{\tilde{Q}_{i}^{t}\right\}_{i=1}^{n_{1}}$ of measure $1 / m$. By re-labeling the $\tilde{k}$ partitions $\left\{\tilde{Q}_{j, s}^{t}\right\}_{j, s} \cup\left\{\tilde{Q}_{i}^{t}\right\}_{i}$, we end up with $\tilde{k}$ partitions $\left\{Q_{i}^{t, m}\right\}_{i=1}^{m}$, for $1 \leq t \leq \tilde{k}$, such that:

1. $\mu_{\mathcal{B}}\left(Q_{i}^{t, m}\right)=1 / m$, for every $i \in\{1, \ldots, m\}, t \in\{1, \ldots, \tilde{k}\}$;

2. $\operatorname{diam}_{d}\left(Q_{i}^{t, m}\right) \leq 1 / r$, if $i>n_{1}$;

3. if $1 \leq i, i^{\prime} \leq n_{1}$ then $Q_{i}^{t, m} \cap Q_{i^{\prime}}^{t^{\prime}, m}=\emptyset$ if $i \neq i^{\prime}$ or $t \neq t^{\prime}$.

Note that the construction of the $k$ partitions $\left\{Q_{i}^{t, m}\right\}_{i=1}^{m}$ was done in such a way that the subsets that do not have small diameters are disjoint, even for different partitions.

Let $\mathbb{M}=\{m(r), r \geq 1\}$ and for every $m=m(r) \in \mathbb{M}$ let $k(m)=\tilde{k}(r)$ as defined above and, for $i, t, m$, let $q_{i}^{t, m}=E_{\mathcal{B}}\left(Q_{i}^{t, m}\right)$. The set $\mathbb{M}$ is unbounded because the 
measure $\mu_{\mathcal{B}}$ being diffuse makes $\lim _{r \rightarrow \infty} m(r)=\infty$, and so $\lim _{r \rightarrow \infty} \tilde{k}(r)=\infty$. For each $t=1, \ldots, k,\left\{q_{i}^{t, m}\right\}_{i=1}^{m} \subset \mathcal{B}^{\prime} \cap \mathcal{M}$ is a partition of the unity.

Let $b \in \mathcal{B}, \epsilon>0$, and let $f \in C(\Gamma(\mathcal{B}))$ be such that $b=\int_{\Gamma(\mathcal{B})} f d E_{\mathcal{B}}$. Then, by compactness, there exists $\delta>0$ such that if $Q \subseteq \Gamma(\mathcal{B})$ with $\operatorname{diam}_{d}(Q)<\delta$ then $\operatorname{diam}(f(Q))<\epsilon$. Let $r \in \mathbb{N}$ be such that $1 / r<\delta$ and $2\|b\| / k(r) \leq \epsilon$; let $m=m(r) \in \mathbb{M}$, and let $\beta_{i}^{t, m}=m \tau\left(b q_{i}^{t, m}\right)=m \int_{Q_{i}^{t, m}} f d \mu_{\mathcal{B}}$. Properties 1-3 translate then into

1'. $\tau\left(q_{i}^{t, m}\right)=1 / m$, for every $i \in\{1, \ldots, m\}, t \in\{1, \ldots, k\}$;

2 '. if $i>n_{1}$, then $\left|f(x)-\beta_{i}^{t, m}\right| \leq \epsilon, \forall x \in Q_{i}^{t, m}$;

3'. if $1 \leq i, i^{\prime} \leq n_{1}$ then $q_{i}^{t, m} \perp q_{i}^{t^{\prime}, m}$ if $i \neq i^{\prime}$ or $t \neq t^{\prime}$.

Therefore we have

$$
\begin{aligned}
\left\|b-\frac{1}{k} \sum_{t=1}^{k} \sum_{i=1}^{m} \beta_{i}^{t, m} q_{i}^{t, m}\right\| & =\left\|\frac{1}{k} \sum_{t=1}^{k}\left(b-\sum_{i=1}^{m} \beta_{i}^{t, m} q_{i}^{t, m}\right)\right\| \\
& =\left\|\frac{1}{k} \sum_{t=1}^{k} \sum_{i=1}^{m} \int_{Q_{i}^{t, m}}\left(f-\beta_{i}^{t, m}\right) d E_{\mathcal{B}}\right\| \\
& \leq\left\|\frac{1}{k} \sum_{t=1}^{k} \sum_{i=1}^{n_{1}} \int_{Q_{i}^{t, m}}\left(f-\beta_{i}^{t, m}\right) d E_{\mathcal{B}}\right\|+\epsilon \\
& \leq\left\|\frac{2\|b\|}{k} \sum_{t=1}^{k} \sum_{i=1}^{n_{1}} q_{i}^{t, m}\right\|+\epsilon \\
& =\frac{2\|b\|}{k}+\epsilon \leq 2 \epsilon
\end{aligned}
$$

where the first inequality is a consequence of 2 ' and the last equality follows from $3^{\prime}$.

Proof of Lemma 3.6. Fix a norm dense subset $B=\left(b_{j}\right)_{j \in \mathbb{N}} \subseteq \mathcal{B}$. In the construction leading to Dixmier's Theorem, a previous result [20, 8.3.4] asserts that for each $j$, there exists a sequence $\left\{\rho_{j}^{n}\right\}_{n \in \mathbb{N}} \subseteq \mathcal{D}(\mathcal{M})$ such that for every $1 \leq h \leq j, \| \rho_{j}^{n}\left(b_{h}\right)-$ $\tau\left(b_{h}\right) I \| \stackrel{n}{\rightarrow} 0$. For each $j \in \mathbb{N}$, let $n_{0}=n_{0}(j) \in \mathbb{N}$ be such that if $n \geq n_{0}$ then $\left\|\rho_{j}^{n}\left(b_{h}\right)-\tau\left(b_{h}\right) I\right\| \leq 1 / j$ for $1 \leq h \leq j$. If we let $\rho_{j}=\rho_{j}^{n_{0}(j)}$ for $j \in \mathbb{N}$, we get $\left\|\rho_{j}\left(b_{h}\right)-\tau\left(b_{h}\right) I\right\| \stackrel{j}{\rightarrow} 0$ for every $h \in \mathbb{N}$. Since $\left(b_{j}\right)_{j \in \mathbb{N}}$ is norm dense in $\mathcal{B}$ we have $\lim _{j}\left\|\rho_{j}(b)-\tau(b) I\right\|=0$ for every $b \in \mathcal{B}$.

For every $i=1, \ldots, m$, consider the factor $p_{i} \mathcal{M} p_{i}$ with (normalized) trace $\tau_{i}\left(p_{i} x\right)=$ $\tau\left(x p_{i}\right) / \tau\left(p_{i}\right)$. By the Dixmier approximation property mentioned in the first paragraph, applied to the separable $C^{*}$-subalgebra $p_{i} \mathcal{B}$ of the finite factor $p_{i} \mathcal{M} p_{i}$, there exists a sequence $\left\{\rho_{j}^{i}\right\}_{j \in \mathbb{N}} \in \mathcal{D}\left(p_{i} \mathcal{M} p_{i}\right)$ such that $\lim _{j \rightarrow \infty}\left\|\rho_{j}^{i}\left(p_{i} b\right)-\tau_{i}\left(p_{i} b\right) p_{i}\right\|=0$, for every $b \in \mathcal{B}$.

For each $\rho \in \mathcal{D}\left(p_{i} \mathcal{M} p_{i}\right)$, we can consider an extension $\tilde{\rho} \in \mathcal{D}(\mathcal{M})$ as follows: if $\rho\left(p_{i} b\right)=\sum_{h=1}^{k} \lambda_{h} u_{h} b u_{h}^{*}$, with $u_{h} \in \mathcal{U}\left(p_{i} \mathcal{M} p_{i}\right)$, define $\tilde{\rho} \in \mathcal{D}(\mathcal{M})$ by $\tilde{\rho}(b)=$ $\sum_{h=1}^{k} \lambda_{h} \tilde{u}_{h} b \tilde{u}_{h}^{*}$, where $\tilde{u}_{h}=u_{h}+\left(1-p_{i}\right) \in \mathcal{U}(\mathcal{M})$. If $1 \leq i \leq m$ set $\rho_{j}=\prod_{i=1}^{m} \tilde{\rho}_{j}^{i}$ 
for $j \geq 1$. It is easy to verify that if $1 \leq i \leq m$ then $\rho_{j}\left(b p_{i}\right)=\tilde{\rho}_{j}^{i}\left(b p_{i}\right)$ for every $b \in \mathcal{B}$. Then, if $b \in \mathcal{B}$,

$$
\left\|\rho_{j}(b)-\sum_{i=1}^{m} \beta_{i}(b) p_{i}\right\|=\left\|\sum_{i=1}^{m} \tilde{\rho}_{j}^{i}\left(b p_{i}\right)-\tau_{i}\left(b p_{i}\right) p_{i}\right\| \underset{j \rightarrow \infty}{\underset{\longrightarrow}{\longrightarrow}} 0 .
$$

Ackowledgements. We wish to thank Professors D. Farenick and D. Stojanoff for their support and useful discussions regarding the material in this paper.

\section{REFERENCES}

[1] P.M. Alberti and A. Uhlmann, Stochasticity and partial order. Doubly stochastic maps and unitary mixing. Mathematische Monographien, 18. VEB Deutscher Verlag der Wissenschaften, Berlin, 1981.

[2] P.M. Alberti, and A. Uhlmann, Dissipative motion in state spaces. Teubner-Texte zur Mathematik, 33. BSB B. G. Teubner Verlagsgesellschaft, Leipzig, 1981.

[3] E.M. Alfsen,Compact Convex Set and Boundary Integrals, Springer-Verlag, New York, NY 1971.

[4] T. Ando, Majorization, doubly stochastic matrices and comparison of eigenvalues, Lecture Notes, Hokkaido Univ., 1982.

[5] J. Antezana, P. Massey, and D. Stojanoff, Jensen's Inequality and Majorization, J. Math. Anal. App. 3311 (2007), 297-307.

[6] J. Antezana, P. Massey, M. Ruiz and D. Stojanoff, The Schur-Horn theorem for operators and frames with prescribed norms and frame operator, Illinois J. of Math. 512 (2007), 537-560.

[7] M. Argerami and P. Massey, A Schur-Horn Theorem in II factors, Indiana Univ. Math. J., 56 No. 5 (2007), 2051-2060

[8] M. Argerami and P. Massey, A contractive version of a Schur-Horn theorem in II factors, J. Math. Anal. App., 337, 1 (2008), 231-238.

[9] W. Arveson, Subalgebras of $C^{*}$-algebras Acta Math. 123 (1969), 141-224.

[10] W. Arveson and R. Kadison, Diagonals of self-adjoint operators, In D. R. Larson D. Han, P. E. T. Jorgensen, editor, Operator theory, operator algebras and applications, Contemp. Math. Amer. Math. Soc., 2006.

[11] G. Birkhoff, Three observations on linear algebra. (Spanish) Univ. Nac. Tucumán. Revista A. 5, (1946). 147-151.

[12] J.B. Conway, A course in functional analysis, Springer-Verlag, New York, NY 1990.

[13] T. Fack, Sur la notion de valeur caractéristique, J. Operator Theory (1982), 307-333.

[14] D.R. Farenick and S.M. Manjegani, Young's Inequality in Operator Algebras, J. Ramanujan Math. Soc. 20 (2005), no. 2, 107-124. http://xxx.lanl.gov/abs/math.0A/0303318.

[15] F. Hiai, Majorization and Stochastic maps in von Neumann algebras, J. Math. Anal. Appl. 127 (1987), no. 1, 18-48.

[16] F. Hiai, Spectral majorization between normal operators in von Neumann algebras, Operator algebras and operator theory (Craiova, 1989), 78-115, Pitman Res. Notes Math. Ser., 271, Longman Sci. Tech., Harlow, 1992.

[17] F. Hiai and Y. Nakamura, Closed Convex Hulls of Unitary Orbits in von Neumann Algebras, Trans. Amer. Math. Soc. 323 (1991), 1-38.

[18] R. Kadison, The Pythagorean theorem I: the finite case, Proc. N.A.S. (USA), 99(7):4178-4184, 2002.

[19] R. Kadison, The Pythagorean theorem II: the infinite discrete case, Proc. N.A.S. (USA), 99(8):5217-5222, 2002.

[20] Kadison and Ringrose, Fundamentals of the Theory of Operator Algebras, Vol II, Academic Press, Orlando, Florida, 1986.

[21] E. Kamei, Majorization in finite factors, Math. Japonica 28, No. 4 (1983), 495-499.

[22] F.D. Martínez Pería, P. Massey, L. Silvestre, Weak matrix majorization. Linear Algebra Appl. 403 (2005), 343-368. 
[23] P. Massey, Refinements of spectral resolutions and modelling of operators in $I_{1}$ factors, J. Op. Th., to appear.

[24] A. Neumann, An infinite-dimensional version of the Schur-Horn convexity theorem, J. Funct. Anal. 161 (1999), 418-451.

[25] W. Rudin, Real and complex analysis. Third edition. McGraw-Hill Book Co., New York, 1987.

[26] D. Sherman, Unitary orbits of normal operators in von Neumann algebras, Journal fur die Reine und Angewandte Mathematik 605 (2007), 95-132.

[27] M. Takesaki, Theory of Operator Algebras I, Encyclopaedia of Mathematical Sciences V, Springer Verlag, 2nd printing of the First Edition 1979.

Department of Mathematics and Statistics, University of Regina, Saskatchewan, CANADA S4S 0A2, argerami@math.uregina.ca

Departamento de Matemática, Facultad de Ciencias Exactas, Universidad Nacional DE La Plata, Argentina, massey@mate.unlp.edu.ar 